\title{
Originals
}

\section{Genetic influence of the R/Q353 genotype on factor VII activity is overwhelmed by environmental factors in Chinese patients with Type II (non-insulin-dependent) diabetes mellitus}

\author{
K.S.L. Lam ${ }^{1}$, O.C.K. Ma ${ }^{2}$, C. Bourke ${ }^{3}$, L.C. Chan ${ }^{3}$, E. D. Janus ${ }^{2}$ \\ ${ }^{1}$ Department of Medicine, University of Hong Kong, Queen Mary Hospital, Hong Kong \\ ${ }^{2}$ Department of Pathology, University of Hong Kong, Queen Mary Hospital, Hong Kong \\ ${ }^{3}$ Clinical Biochemistry Unit, University of Hong Kong, Queen Mary Hospital, Hong Kong
}

\begin{abstract}
Summary We studied the effects of genetic and environmental influences on factor VII coagulant activity (VIIc) in Chinese diabetic patients (263 with Type II [non-insulin-dependent] diabetes mellitus, 78 with Type I [insulin-dependent] diabetes mellitus) and 143 normal control subjects. VIIc was measured by a one-stage biological assay. The R/Q353 or Msp1 polymorphism at codon 353 of the factor VII gene was detected after Msp1 digestion of polymerase chain reaction-amplified genomic DNA. In both diabetic and control subjects the allele frequencies of the R (M1) and Q (M2) alleles were 0.96 and 0.04; the corresponding reported frequencies in Caucasians being 0.90 and 0.10: VIIc were $21 \%$ lower in Chinese control subjects and Type I diabetic patients with $\mathrm{R} / \mathrm{Q}$, compared with $\mathrm{R} / \mathrm{R}$ subjects $(p<0.001$ and $p<0.05)$. The corresponding difference was $4 \%$ for Type II diabetc patients ( $p=$ NS). Type II diabetic patients had higher mean VIIc levels than control subjects and Type I diabetic patients $(p<0.01)$; they were also older, and had higher serum creatinine and triglyceride (all $p<0.01$ ). They also had higher VIIc levels than an age-matched older control group $(p<0.01 ; n=182)$ in whom the genotype effect was
\end{abstract}

clearly seen. On stepwise linear regression analysis, the significant independent determinants of VIIc were serum triglyceride (contributing $20 \%$ and $25 \%$ to variance in control subjects and diabetic patients), the R/Q353 genotype (contributing to $12 \%$ of the variance in control subjects but only $1 \%$ in diabetic patients), age and total cholesterol in all subjects, and in the diabetic patients female sex, urinary albumin excretion rate and serum creatinine. VIIc was higher in diabetic patients with macroangiopathy and retinopathy (both $p<0.0001$ ). We conclude that compared with Caucasians, the $\mathrm{Q}$ allele frequency is significantly lower in these Chinese subjects. Plasma VIIc is determined by both genetic and environmental influences such that in Chinese Type II diabetic patients, the effect of environmental factors predominates, almost negating the influence of the R/Q353 genotype. High VIIc may contribute to the increased cardiovascular risk in Type II diabetic patients. [Diabetologia (1998) 41: 760-766]

Keywords Factor VII, diabetes mellitus, genetic, environmental, Chinese.
Received: 24 October 1997 and in final revised form: 28 January 1998

Corresponding author: Professor K. Lam, Department of Medicine, University of Hong Kong, Queen Mary Hospital, Pokfulam Road, Hong Kong

Abbreviations: VIIc, Factor VII coagulant activity; apo, apolipoprotein; MAER, mean albumin excretion rate; PCR, polymerase chain reaction.
Diabetes mellitus is associated with a two to threefold increase in cardiovascular mortality [1]. The mechanism whereby diabetes increases cardiovascular risk is probably multifactorial. Recent studies suggest that this may in part be attributed to an increase in thrombotic tendency [2] including an increase in circulating level of factor VII, a key enzyme in the initiation of coagulation. A positive correlation between increased factor VII activity and cardiovascular mortality has been shown in the Northwick Park Heart Study [3]. A similar trend 
has also been demonstrated in the PROCAM study [4].

Several genetic variations of the factor VII gene affecting the promotor region [5], intron 7 [6] and exon 8 [7], respectively, have been reported to contribute, to different extents [8], to variance in VIIc levels in the general population. Of these, the best studied is the R/Q353 or Msp1 polymorphism in exon 8: a guanine $(G)$ to adenine $(A)$ substitution in codon 353 leads to the loss of a cut site by the restriction enzyme Msp1 and the replacement of arginine (R353 or M1 allele) by glutamine (Q353 or M2 allele) in the protein product. Carriers of the $\mathrm{Q}$ allele have been found consistently to possess levels of VIIc $15-30 \%[7,8,9]$ lower than those homozygous for the $\mathrm{R}$ allele in various ethnic populations. The $\mathrm{Q}$ allele frequency, on the other hand, differs considerably among the ethnic groups studied: from as low as $3.4 \%$ in Japanese [9] to as high as $29 \%$ in Dravidian Indians [10] and Alberta Hutterites [11].

In addition to genetic influence, circulating levels of VIIc are also closely related to several environmental factors. Women have higher VIIc levels than men $[12,13]$. A positive correlation has been demonstrated between factor VII activity and serum levels of total triglyceride and/or cholesterol [7, 9, 10, 12, 14], age and body mass index [12]. In some studies the correlation between plasma triglyceride and VIIc was found to be genotype specific [9, 10, 15-17] suggesting an interaction between genetic and environmental influence. A positive correlation between fasting plasma glucose and factor VII level has also been reported [18], and VIIc levels are increased in patients with Type II diabetes mellitus especially in the presence of microalbuminuria or albuminuria [19]. In a study of 95 Caucasian Type II diabetic patients, the R/Q353 genotype was found to contribute to $12 \%$ of the variance in VIIc levels. Although direct comparison with a control group was not included in this study, the genetic contribution was apparently lower than that generally quoted for Caucasians [7], and may reflect the effects of environmental factors such as hyperlipidaemia and other metabolic disturbances in these patients.

The allele frequency of the Q allele and its effect on plasma VIIc have not been previously reported in Chinese subjects. In this study, the effects of the R/ Q353 genotype and various environmental factors on circulating VIIc were investigated in 666 Chinese subjects to determine the relative importance of genetic and environmental influences on VIIc levels in the southern Chinese and whether this is altered by the presence of diabetes mellitus.

\section{Subjects and methods}

Subjects. Table 1 shows the characteristics of non-diabetic and diabetic subjects. All were southern Chinese. Control subjects were unrelated healthy volunteer hospital staff ( 51 men, 92 women). Their mean age was within the peak age of the Hong Kong adult population (35-39 years), according to the Government Census in 1996. To provide an age-matched older control group for the Type II diabetic patients, data from 182 nondiabetic subjects, (40-74 years), who were among the first 500 subjects to be recruited randomly into the age-stratified, population-based Hong Kong Cardiovascular Risk Factor Prevalence Study (1995-1996; principal investigator: E.D.Janus) were also included for analysis. Data on serum creatinine, liver function, $\mathrm{HbA}_{1 \mathrm{c}}$, apolipoprotein $\mathrm{A} 1$ and $\mathrm{B}$ were not available in this older control group. The diabetic subjects were recruited from the Diabetes Clinic of the University Department of Medicine, Queen Mary Hospital, and consisted of 263 Type II diabetic patients (129 men and 134 women) and 78 patients with insulin-dependent diabetes (Type I; 31 men and 47 women). All Type II diabetic patients could be controlled on diet with or without oral drugs for at least one year prior to requirement of insulin therapy. The Type I diabetic patients had severe symptoms of diabetes in association with ketosis or ketoacidosis and had required insulin therapy since diagnosis; c-peptide concentrations, available in over half of them, confirmed significant insulin deficiency. All gave informed consent and the protocol was approved by the ethics committee of the Medical Faculty, University of Hong Kong. All were studied after overnight fasting. Except for the older control subjects, all had registered as normal in a liver function test. Clinical data were obtained from the clinic records and confirmed by a direct interview, physical examination and an electrocardiogram performed on the study day. Smokers referred to current smokers and those who had been smoking up to 3 months prior to the study. Ischaemic heart disease was diagnosed by a history of myocardial infarction, angina or evidence of myocardial infarction (pathological Q-wave) or significant horizontal ST segment depression ( $\geq 2 \mathrm{~mm}$ ) in a 12-lead resting electrocardiogram. Macrovascular disease included ischaemic heart disease, stroke, transient ischaemic attack or peripheral vascular disease, as evidenced by intermittent claudication, rest pain, limb amputation or arterial surgery. Blood pressure was measured with a standard sphygmomanometer after 10 min of rest in the sitting position. Retinopathy was determined by ophthalmoscopy (with pupils dilated to $>3 \mathrm{~mm}$ diameter). Peripheral neuropathy was assessed by means of the vibration perception threshold determined using a biothesiometer. Microalbuminuria or albuminuria was assessed from the mean albumin excretion rate (MAER), based on two consecutive 12-h overnight urine collections.

DNA analysis. DNA was extracted from white blood cells in 5-10 ml EDTA blood using standard "SDS/proteinase K" lysis and phenol/chloroform purification methods. A $317 \mathrm{bp}$ portion of the factor VII gene was amplified by polymerase chain reaction (PCR) as described [7] using $100 \mathrm{ng}$ of genomic DNA, $1 \mathrm{U}$ of thermostable Taq polymerase (Perkin-Elmer Cetus, Branchburg, N.J., USA) and PCR buffer containing $1.5 \mathrm{mmol} / 1 \mathrm{MgCl}_{2}$. The sequences of the primers $\left(5^{\prime}-3^{\prime}\right)$ were GGG AGA CTC CCC AAA TAT CAC and ACG CAG CCT TGG CTT TCT CTC. PCR reactions were performed on a Perkin-Elmer DNA cycler (model 480 Branchburg, USA) for 30 cycles, each with a denaturation step of $1 \mathrm{~min}$ at $95^{\circ} \mathrm{C}$, annealing for $1 \mathrm{~min}$ at $60^{\circ} \mathrm{C}$ and extension for $1 \mathrm{~min}$ at $72^{\circ} \mathrm{C}$. The PCR product was digested with $10 \mathrm{U}$ Msp1 (New England Biolabs, Beverley, Mass., USA) at $37^{\circ} \mathrm{C}$ overnight. 
Table 1. Study group characteristics

\begin{tabular}{|c|c|c|c|c|}
\hline & $\begin{array}{l}\text { Control subjects } \\
(n=143)\end{array}$ & $\begin{array}{l}\text { Type I } \\
\text { diabetes } \\
(n=78)\end{array}$ & $\begin{array}{l}\text { Type II } \\
\text { diabetes } \\
(n=263)\end{array}$ & $\begin{array}{l}\text { Older } \\
\text { control subjects } \\
(n=182)\end{array}$ \\
\hline Age (years) & $37.0 \pm 10.2$ & $35.5 \pm 11.1^{\mathrm{c}}$ & $52.3 \pm 16.2^{\mathrm{b}}$ & $51.6 \pm 9.0^{\mathrm{bf}}$ \\
\hline $\operatorname{Sex}(M / F)$ & $51 / 92$ & $31 / 47$ & $129 / 134^{\mathrm{b}}$ & $90 / 92^{\mathrm{a}}$ \\
\hline SMO (\%) & 6 & $5^{\mathrm{c}}$ & $14^{\mathrm{a}}$ & $21^{\mathrm{bcf}}$ \\
\hline BMI & $23.6 \pm 4.7$ & $21.6 \pm 2.6$ & $24.8 \pm 4.4$ & $23.9 \pm 3.1$ \\
\hline Insulin therapy (\%) & - & $100^{\mathrm{d}}$ & 52 & - \\
\hline Insulin dose (units/D) & - & $39.5 \pm 12.9$ & $40.1 \pm 17.4$ & - \\
\hline Diabetes duration (years) & - & $11.7 \pm 6.2^{\mathrm{c}}$ & $9.7 \pm 6.9$ & - \\
\hline Macrovascular disease (\%) & - & $12^{\mathrm{d}}$ & 36 & - \\
\hline Ischaemic heart disease (\%) & - & $5^{\mathrm{d}}$ & 21 & - \\
\hline Stroke $(\%)$ & - & 3 & 5 & - \\
\hline Peripheral neuropathy (\%) & - & $6^{\mathrm{d}}$ & 19 & - \\
\hline Retinopathy (\%) & - & $9^{\mathrm{d}}$ & 25 & - \\
\hline \multicolumn{5}{|c|}{ Factor VII genotype and coagulant activity (VIIc), plasma lipids and other biochemical parameters } \\
\hline Genotype $(\%)^{\mathrm{g}}(\mathrm{R} / \mathrm{R}: \mathrm{R} / \mathrm{Q}: \mathrm{Q} / \mathrm{Q})$ & 126:12:0 & $67: 5: 1$ & 230:17:1 & $167: 15: 0$ \\
\hline VIIc $(\%)^{g}$ & $113.4 \pm 23.8^{\mathrm{d}}[118.3]^{\mathrm{d}}$ & $113.6 \pm 33.9^{\mathrm{c}}[119.6]^{\mathrm{c}}$ & $130.1 \pm 38.5[130.3]$ & $118.6 \pm 20.5^{\mathrm{ad}}[115.8]^{\mathrm{d}}$ \\
\hline Total cholesterol (mmol/l) & $5.1 \pm 0.9$ & $5.0 \pm 0.9$ & $5.3 \pm 1.1$ & $5.3 \pm 1.0$ \\
\hline Triglyceride $(\mathrm{mmol} / \mathrm{l})$ & $1.1 \pm 0.7^{\mathrm{df}}$ & $0.7 \pm 0.5^{\mathrm{d}}$ & $2.1 \pm 2.3$ & $1.1 \pm 0.6^{\mathrm{df}}$ \\
\hline HDL-cholesterol (mmol/l) & $1.3 \pm 0.3^{\mathrm{df}}$ & $1.7 \pm 0.5^{\mathrm{d}}$ & $1.1 \pm 0.4$ & $1.4 \pm 0.4^{\mathrm{df}}$ \\
\hline LDL-cholesterol (mmol/l) & $3.3 \pm 0.8^{\mathrm{e}}$ & $3.0 \pm 0.7$ & $3.3 \pm 1.0^{\mathrm{e}}$ & $3.4 \pm 0.9^{f}$ \\
\hline Apo $A_{1}(\mathrm{mg} / \mathrm{dl})$ & $1.5 \pm 0.3$ & $1.6 \pm 0.3^{\mathrm{d}}$ & $1.4 \pm 0.2^{\mathrm{b}}$ & - \\
\hline Apo B (mg/dl) & $1.1 \pm 0.3$ & $1.0 \pm 0.3^{\mathrm{d}}$ & $1.3 \pm 0.3^{b}$ & - \\
\hline $\mathrm{HbA}_{1 \mathrm{c}}(\%)$ & $4.1 \pm 0.6$ & $6.9 \pm 1.7^{\mathrm{bc}}$ & $7.4 \pm 2.2^{\mathrm{b}}$ & - \\
\hline Plasma creatinine $(\mathrm{mol} / \mathrm{l})$ & $80.8 \pm 15.0$ & $89.8 \pm 53.9^{c}$ & $108.9 \pm 87.0^{\mathrm{b}}$ & - \\
\hline $\operatorname{MAER}(\mu \mathrm{g} / \mathrm{min})$ & - & $46.6 \pm 168.3^{\mathrm{d}}$ & $209.4 \pm 611.7$ & - \\
\hline
\end{tabular}

Means $\pm \mathrm{SD} ;[\chi]$, age-adjusted mean values

a, $p<0.05 ; \mathrm{b}, p<0.01$ vs control subjects

c, $p<0.05 ; \mathrm{d}, p<0.01$ vs Type II diabetes

e, $p<0.05$; $\mathrm{f}, p<0.01$ vs Type I diabetes

DNA fragments were separated on a $3 \%$ agarose gel at $80 \mathrm{~V}$ for $2 \mathrm{~h}$ and visualised by staining with $0.5 \mu \mathrm{g} / \mathrm{ml}$ ethidium bromide and photographed under ultraviolet light for genotyping.

Assays. VIIc was measured in trisodium citrate samples by a one-stage biological assay using ACL 1000 (Instrumentation Laboratory, Warrington, UK), and was assayed by the amount of prothrombin time corrected by the test plasma when mixed with Factor VII deficient plasma (Bio Merieux SA; Marayl'Etoile, France). The thromboplastin used was supplied by Behring (Marberg, Germany). VIIc was expressed as a percentage of the activity given by a reference plasma (Immuno AG, Vienna, Austria). Coefficients of variation were $3.1 \%$ within run and $3.3 \%$ between runs. Two dilutions were tested for each patient.

In all subjects, $10 \mathrm{ml}$ EDTA blood was taken after fasting for $10-12 \mathrm{~h}$ for the measurement of $\mathrm{HbA}_{1 \mathrm{c}}$ and lipoproteins. Plasma creatinine was measured in heparinised blood. $\mathrm{HbA}_{1 \mathrm{c}}$ was measured by electrophoresis using Beckman Paragon Diatric $\mathrm{HbA}_{1 \mathrm{c}}$ kits (Beckman Instruments, Brea, Calif., USA). Total cholesterol and triglyceride were determined enzymatically (Boehringer Mannheim, Mannheim, Germany) on a Hitachi 717 analyser. HDL cholesterol was measured after VLDL and LDL precipitation with polyethylene glycol 6000. LDL cholesterol was calculated by Friedeward equation. Apolipoprotein A1 (apo A1) and B (apo B), and creatinine were measured nephelometrically on the Beckman Array system (Beckman Instruments). Plasma creatinine was measured by the Jaffe reaction.

Statistical analysis. Statistical analysis was performed using the SPSS (version 4 Chicago, USA) statistics package. Student's $t$ - no. of subjects studies was $138,248,73$ and 182 for control subjects, Type II diabetic patients, Type I diabetic patients and older control subjects respectively.

SMO, smokers; BMI, body mass index test and chi-squares were used for comparison of continuous and categoric variables respectively. Analysis of variance followed by Duncan's multiple range test was used for comparison of multiple groups. Values for VIIc and triglyceride were not normally distributed and were log-transformed prior to analysis. As indicated, VIIc levels were adjusted for confounding variables such as age, triglyceride and cholesterol levels prior to between-group comparisons. Correlation between variables were tested using Pearson's correlation analysis. Only those parameters showing associations with VIIc with $p$ values less than 0.1 on univariate analysis were further analysed using stepwise multiple linear regression to determine whether they contributed independently to changes in circulating VIIc levels. Data are expressed as means \pm SD.

\section{Results}

The frequency distribution of the R/Q353 genotypes was similar in the four groups of subjects (Table 1). About $90 \%$ of the subjects were homozygous for the $\mathrm{R}$ allele and only two subjects were homozygous for the $\mathrm{Q}$ allele (one each in the two patient groups). The overall frequency of the Q allele was $4.1 \%$.

Table 2 shows the mean VIIc values, with and without adjustment for the effects of age, triglyceride and total cholesterol. Among the control subjects those with the R/Q genotype had a mean VIIc activity which was $21 \%$ lower than that of the R/R subjects 
Table 2. Factor VII coagulant activity (\% of reference plasma) in control subjects and patients with different genotypes

\begin{tabular}{lll}
\hline & $\mathrm{R} / \mathrm{R}$ & $\mathrm{R} / \mathrm{Q}$ \\
\hline $\begin{array}{lll}\text { Control subjects } \\
(n=138)\end{array}$ & $115.7 \pm 22.9[115.5]$ & $91.3 \pm 21.5[93.4]^{\mathrm{c}}$ \\
$\quad$ Men $(n=49)$ & $116.1 \pm 24.9[115.0]$ & $89.7 \pm 16.2[97.6]^{\mathrm{b}}$ \\
$\quad$ Women $(n=89)$ & $115.5 \pm 21.9[115.8]$ & $93.0 \pm 27.3[89.5]^{\mathrm{b}}$ \\
$\begin{array}{l}\text { Older Control subjects } \\
(n=182)\end{array}$ & $119.5 \pm 19.6[119.5]$ & $108.1 \pm 27.9[107.8]^{\mathrm{b}}$ \\
$\quad \begin{array}{l}\text { Men }(n=90) \\
\quad \text { Women }(n=92)\end{array}$ & $117.4 \pm 19.8[117.4]$ & $107.2 \pm 19.7[107.2]$ \\
$\begin{array}{l}\text { Type II diabetes } \\
(n=247)\end{array}$ & $121.6 \pm 19.2[121.7]$ & $108.8 \pm 33.5[107.9]^{\mathrm{b}}$ \\
$\begin{array}{l}\text { Type I diabetes } \\
(n=72)\end{array}$ & $130.3 \pm 38.8[130.3]$ & $128.8 \pm 35.4[127.9]$ \\
\hline
\end{tabular}

Means $\pm \mathrm{SD} ;[\chi]$, mean values adjusted for effects of age, triglyceride and total cholesterol

$\mathrm{a}, p<0.05 ; \mathrm{b}, p<0.01 ; \mathrm{c}, p<0.001$ vs $\mathrm{R} / \mathrm{R}$

Table 3. Stepwise linear regression models with factor VII coagulant activity as the dependent variable

\begin{tabular}{llc}
\hline Model & $R^{2}$ & $p$-value \\
\hline Control subjects & & \\
TG & 0.205 & $<0.001$ \\
TG, R/Q & 0.322 & $<0.001$ \\
TG, R/Q, Age & 0.379 & $<0.005$ \\
TG, R/Q, Age, TC & 0.406 & $<0.005$ \\
Diabetic Patients (Type II + Type I) & & \\
TG & 0.251 & $<0.001$ \\
TG, Age & 0.290 & $<0.001$ \\
TG, Age, sex & 0.325 & $<0.001$ \\
TG, Age, sex, MAER & 0.347 & $<0.02$ \\
TG, Age, sex, MAER, TC & 0.361 & $<0.01$ \\
TG, Age, sex, MAER, TC, R/Q & 0.372 & $<0.01$ \\
TG, Age, sex, MAER, TC, R/Q, SCr & 0.381 & $<0.02$ \\
\hline TG, log triglyceride, MAER, mean
\end{tabular}

$\mathrm{TG}, \log$ triglyceride; MAER, mean albumin excretion rate; TC, total cholesterol; R/Q, R/Q353 genotype; SCr, serum creatinine

$(p<0.001)$. This difference in VIIc between the 2 genotypes was statistically significant in the women $(p<0.001)$ and was of borderline significance for the men $(p=0.055)$. VIIc levels in the older control group were also lower in the $\mathrm{R} / \mathrm{Q}$ subjects $(p<0.01)$ and again, the difference was significant only in the women $(p<0.01)$. Among the diabetic patients, the differences in VIIc between $\mathrm{R} / \mathrm{R}$ and $\mathrm{R} / \mathrm{Q}$ subjects were significant in the Type I diabetic patients $(p<0.05)$ but not in the Type II diabetic patients $(p=0.236)$.

VIIc activity were similar between control subjects and type I diabetic patients (Table 1) but were considerably higher among the Type II diabetic patients $(p<0.01$ compared with control subjects or type I diabetic patients even when age-adjusted). Type II diabetic patients were older and consisted of more smokers than control subjects and Type I diabetic patients (Table 1). They also had higher triglyceride, apo $\mathrm{B}, \mathrm{HbA}_{1 \mathrm{c}}$ and plasma creatinine, and lower
HDL cholesterol and apo A1, than the other two groups (Table 1). Compared with control subjects, there were more men in the Type II diabetic group $(p<0.01)$. Type I diabetic patients had higher $\mathrm{HbA}_{1 \mathrm{c}}$ and HDL cholesterol $(p<0.01)$, and lower LDL cholesterol levels $(p<0.05)$ than control subjects. Even when compared with the sex and age matched older control subjects, Type II diabetic patients had higher VIIc and serum triglyceride, and lower HDL-cholestrol levels (all $p<0.01$; Table 1). The difference in VIIc between control subjects and older control subjects $(p<0.05)$ disappeared after adjusting for the effect of age.

Among the diabetic patients, VIIc levels were higher among those with macrovascular disease $(137.2 \pm 28.9 \%$ vs no macrovascular disease: $125.3 \pm 34.3 \% ; \quad p<0.0001)$, retinopathy $(142.5 \pm$ $34.3 \%$ vs no retinopathy: $125.1 \pm 32.0 \% ; p<0.0001)$ and peripheral neuropathy $(136.3 \pm 28.3 \%$ vs no neuropathy: $127.3 \pm 33.9 \% ; p<0.05)$. The prevalence of macrovascular disease, ischaemic heart disease, peripheral neuropathy and retinopathy were all significantly higher in Type II diabetic patients $(p<0.01$ vs Type I diabetic patients; Table 1$)$. They also had higher serum creatinine and MAER $(p<0.05$ and $p<0.01$ vs type I diabetic respectively; Table 1$)$.

When the data from all subjects $(n=666)$ were analysed, women had higher VIIc (124.4 $\pm 28.9 \%)$ than men $(119.2 \pm 28.1 \%)(p<0.02)$. Among the non-diabetic subjects (controls + older controls), a strong correlation between log VIIc and log triglyceride levels was found for subjects with the R/R genotype $(\mathrm{r}=0.375, p<0.001 ; n=293)$ but not when the $\mathrm{Q}$ allele was present $(\mathrm{r}=0.262 ; p=0.19 ; n=27)$. Among Type II diabetic patients, the correlation was highly significant in $R / R$ subjects $(r=0.449$, $p<0.001, n=230)$ and was of borderline significance in those with the $\mathrm{Q}$ allele $(\mathrm{r}=0.479, p=0.052$, $n=18)$.

In stepwise linear regression models, log triglyceride, R/Q353 genotype, age and total cholesterol were found to be significant independent predictors of VIIc among control subjects (Table 3), contributing 20,11, 5 and $2 \%$ respectively to the variance in VIIc levels. These were also significant independent predictors of VIIc among the diabetic (Type II and Type I) patients (Table 3). However, while log triglyceride was still the most significant predictor and contributed to $25 \%$ of the variation in VIIc, the contribution of the R/Q353 genotype was reduced to $1 \%$ only among the diabetic patients. Other factors of significant predictive values among the patients included age $(4 \%)$, sex $(4 \%)$, MAER $(2 \%)$, cholesterol $(1 \%)$ and serum creatinine $(1 \%)$. 


\section{Discussion}

In this study, it was found that the R/Q353 or Msp1 genotype of the factor VII gene, an important genetic determinant of factor VII activity among Caucasians $[7,8]$, was also an independent predictor of factor VII expression in this Chinese population. As in Caucasians [7], carriers of the Q allele had factor VII levels which were about $20 \%$ below the mean value of the general population. However, the frequency of the Q allele was only $4.1 \%$, considerably lower than that reported for Caucasians [7] (10\%), Afro-Caribbeans $(8 \%)$ [16], Indians $(25-29 \%)[16,10]$ and Alberta Hutterites $(29 \%)$ [11]. It is interesting to note that a low Q allele frequency $(3.4 \%)$ has also been reported for the Japanese [9], an ethnic group which is more closely related to the Chinese geographically, and perhaps also genetically. The contribution of the R/Q353 genotype to factor VII activity in the Chinese control population was similar to that reported for Indians [10], but considerably lower than that found in studies on Caucasians [7, 8] (20-31\%). The contribution became even less among diabetic patients and was almost negligible in the Type II diabetic patients, probably because of the overwhelming effects of environmental factors, especially hypertriglyceridaemia, impaired renal function and microalbuminuria or albuminuria, which were prevalent in these patients.

Our findings were at variance with those of Heywood et al. [12] who reported that the R/Q353 genotype contributed to $12 \%$ of the variance in VIIc levels in 95 Caucasian Type II diabetic patients. This may be explained in part by ethnic differences as the $\mathrm{Q}$ allele frequency is much higher in Caucasians. Differences in sample characteristics may also contribute to the discrepancy in the two studies. Information on nephropathy status was not included in the study by Heywood et al. [12]. In this study, however, over half of the Type II diabetic patients had either microalbuminuria or albuminuria so that the genotype effect was more likely to be offset by the confounding effects of urinary albumin loss and impaired renal function.

As in Caucasians $[13,20]$, women in this Chinese population had higher factor VII activity compared with men. The effect of the R/Q353 polymorphism, highly significant among the female control subjects, was also less marked in the men after adjusting for the confounding effects of age, triglyceride and total cholesterol concentrations. The association of factor VII activity with age has been reported previously in Caucasian diabetic patients [12], although in the latter study, the association was not significant on multivariate analysis, probably because of the smaller sample size and age range.

Strong associations between factor VII activity and plasma lipid levels have been well established [7,
$9,10,12,14]$. It has been suggested that lipoproteins rich in negatively charged phospholipids activate factor XII which in turn activates factor VII [21]. Previous studies have reported controversial results regarding the interaction between plasma triglyceride and the R/Q353 genotype in the determination of factor VII activity $[9,10,15,17]$. Among the Japanese, it was reported that the correlation between triglyceride and factor VII activity could only be demonstrated in subjects homozygous for the $\mathrm{R}$ allele [9]. In studies on Caucasians [15, 17], a stronger association between triglyceride and factor VII activity was also found in $R / R$ subjects compared with $Q$ carriers. A study on Dravidian Indians, however, reported that the correlation of factor VII activity with triglyceride could only be demonstrated in carriers of the Q allele [10]. The findings among the non-diabetic subjects in this study also suggested a stronger association between factor VII activity and triglyceride levels in Chinese subjects with the R/R genotype. The interaction between the R/Q353 genotype and plasma triglyceride in determining factor VII activity was less obvious in the Type II diabetic patients probably because of the overwhelming effects on factor VII activity of other environmental variables.

Studies conducted among Caucasians have reported repeatedly plasma cholesterol to be an important predictor of factor VII level [7, 12], and in the latter study [12], cholesterol level was actually found to be a stronger independent determinant of factor VII activity than plasma triglyceride, contributing to $30 \%$ of the variance in factor VII activity among 95 Type II diabetic patients. On the other hand, it would appear from our data that triglyceride was the most important predictor of factor VII activity in the Chinese population, accounting for $20 \%$ and $25 \%$ respectively of the variance in factor VII activity in control subjects and diabetic patients. The association of plasma cholesterol with factor VII activity in our population appeared to be far less impressive, contributing to less than $3 \%$ of the variation in factor VII levels between individuals, irrespective of the presence of diabetes. Whether this is related to any ethnic difference in the regulation of factor VII coagulant activity remains to be determined.

Studies documenting urinary albumin excretion rates report mostly that circulating factor VII activity is not increased in Type II or Type I patients with normoalbuminuria [22-24] and repeatedly find a positive correlation between factor VII and albumin excretion rate in Type II $[19,22]$ as well as Type I [22] diabetic patients. Reports are inconsistent as to increased factor VII levels in patients with microalbuminuria [19, 22-24], although factor VII levels are generally found to be increased in the presence of albuminuria [17, 22, 24]. It has been suggested that albuminuria provides a general stimulus for hepatic synthesis, leading to increased production of various 
proteins including lipoproteins and procoagulant factors [25]. In this study, as reflected by the mean urinary albumin excretion rate, the majority of the Type I diabetic patients were either microalbuminuric or normoalbuminuric, while most of the Type II diabetic patients were either albuminuric or microalbuminuric. The raised factor VII levels in the type II diabetic patients in this study was therefore attributable, to a large extent, to the raised triglyceride and cholesterol levels which are often found in microalbuminuric and albuminuric Type II diabetic patients [26], and their higher serum creatinine and urinary albumin excretion rate, all of which are independent predictors of factor VII levels.

The increased factor VII levels in diabetic patients with macrovascular disease in this study were in accordance with observations among non-diabetic Chinese subjects [27]. Together with the association between raised factor VII activity and increased cardiovascular mortality in prospective studies $[3,4]$, these findings suggest that the raised levels of factor VII activities may have contributed, at least in part, to the increased prevalence of macrovascular disease in the Type II diabetic patients. On the other hand, the presence of coronary heart disease in Type II diabetic patients, even if minimal, can itself contribute to elevated levels of VIIc via the rupture of atheromatous plaques and release of tissue factor, leading to activation of the coagulation system [28]. The increase in patients with retinopathy and neuropathy was not unexpected and may not imply any causal relationship as macrovascular and various microvascular complications often coexist in the same patient with diabetes.

This study illustrates clearly that circulating levels of factor VII activity are determined by the balance between genetic and environmental influences. In Type II diabetic patients, especially in the presence of subclinical or clinical nephropathy, the effect of environmental factors predominates, leading to near negation of the genetic influence associated with the R/Q353 genotype.

Acknowledgements. We thank Ms Elaine Pang and nurses of the KK Leung Diabetes Centre for assistance in data collection, staff of the Clinical Biochemistry Unit for laboratory support, Mr. Stanley Yeung for data processing and Ms Karman Yu for secretarial assistance. This study was supported by a CRCG grant from the University of Hong Kong.

\section{References}

1. Head J, Fuller J (1990) International variations in mortality among diabetic patients:WHO multinational study of vascular disease in diabetics. Diabetologia 33: 477-481

2. Ostermann H, van de Loo J (1986) Factors of the hemostatic system in diabetic patients. A survey of controlled studies. Haemostasis 16: 384-416

3. Meade TW, Ruddock V, Stirling Y, Chakrabarti T, Miller GJ (1993) Fibrinolytic activity, clotting factors and long- term incidence of ischaemic heart disease in the Northwick Park Heart Study. Lancet 342: 1076-1079

4. Junker R, Heinrich J, Schulte H, van de Loo J, Assman G (1997) Coagulation factor VII and the risk of coronary heart disease in healthy men. Arterioscler Thromb Vasc Biol 17: 1539-1544

5. Marchetti G, Patracchini P, Papacchini M, Ferrati M, Bernardi $F$ (1993) A polymorphism in the 5 'region of the coagulation factor VII gene (F7) due to an inserted decanucleotide. Hum Genet 90: 575-576

6. Bernardi F, Patracchini P, Gemmati D et al. (1993) Molecular analysis of factor VII deficiency in Italy, a frequenct mutation (FVII Lazio) in a repeated intronic region. Hum Genet 92: 446-450

7. Green F, Kelleher C, Wilkes H, Temple A, Meade TW, Humphries S (1991) A common genetic polymorphism associated with lower coagulation factor VII levels in healthy individuals. Arterioscler Thromb Vasc Biol 11: 540-546

8. Bernardi F, Marchetti G, Pinotti M et al. (1996) Factor VII gene polymorphism contribute about one third of the factor VII level variation in plasma. Arterioscler Thromb Vasc Biol 16: 72-76

9. Kario K, Narita N, Matsuo Tet al. (1995) Genetic determinants of plasma factor VII activity in the Japanese. Thromb Haemost 73: 617-622

10. Saha A, Liu Y, Heng CK, Hong S, Low PS, Tay JSH (1994) Association of factor VII genotype with plasma factor VII activity and antigen levels in healthy Indian adults and interaction with triglycerides. Arterioscler Thromb Vasc Biol 14: 1923-1927

11. Hegele RA, Breckenridge WC, Brunt JH, Connelly PW (1997) Genetic variation in factor VII associated with variation in plasma lipoprotein(a) concentration. Arterioscler Thromb Vasc Biol 17: 1701-1706

12. Heywood DM, Mansfield MW, Grant PJ (1996) Factor VII gene polymorphism, factor VII:C levels and features of insulin resistance in non-insulin-dependent diabetes mellitus. Thromb Haemost 75: 401-406

13. Mansfield MW, Heywood DM, Grant PJ (1996) Sex differences in coagulation and fibrinolysis in white subjects with non-insulin dependent diabetes mellitus. Arterioscler Thromb Vasc Biol 16: 160-164

14. Mennen LI, Schouten EG, Grabbe DE, Kluft C (1996) Coagulation factor VII, dietary fat and blood lipids: review. Thromb Haemost 76: 492-499

15. Humphries S, Temple A, Lane A, Green F, Cooper J, Miller G (1996) Low plasma levels of factor VIIc and antigen are more strongly associated with the 10 base pair promoter $(-323)$ insertion than the glutaminine 353 variant. Thromb Haemost 75: 567-572

16. Lane A, Cruickshank JK, Mitchell J, Henderson A, Humphries S, Green F (1992) Genetic and environmental determinants of factor VII coagulant activity in ethnic groups at differing risk of coronary heart disease. Atherosclerosis 94: 43-50

17. Humphries SE, Lane A, Green FR, Cooper J, Miller GJ (1994) Factor VII coagulant activity and antigen levels in healthy men are determined by interaction between factor VII genotype and plasma triglyceride concentration. Arterioscler Thromb 14: 193-198

18. Ceriello A, Giugliano D, Quatraro A, Dello Russo P, Torella R (1988) Blood glucose may condition factor VII levels in diabetic and normal subjects. Diabetologia 31: 889-891

19. Kario K, Matsuo T, Kobayashi H, Matsuo M, Sakata T, Miyata T (1995) Activation of tissue factor-induced coagulation and endothelial cell dysfunction in non-insulin-de- 
pendent diabetic patients with microalbuminuria. Arterioscler Thromb Vasc Biol 15: 1114-1120

20. Mennen LI, de Maat MPM, Schouten EG et al. (1997) Coagulation factor VII, serum triglycerides and the R/Q353 polymorphism: differences between older men and women. Thromb Haemost 78: 984-986

21. Miller GJ (1994) Lipoproteins and the haemostatic system in atherothrombotic disorders. Baillieres Clin Haematol 7: 713-732

22. Knobl P, Schernthaner, Schnack C et al. (1993) Thrombogenic factors are related to urinary albumin excretion rate in type 1 (insulin-dependent) and type 2 (non-insulin-dependent) diabetic patients. Diabetologia 36: 1045-1050

23. Allawi J, Jarrett RJ (1990) Microalbuminuria and cardiovascular risk factors in type 2 diabetes mellitus. Diabetic Medicine 7: 115-118

24. Lee P, Jenkins A, Bourke C et al. (1993) Prothrombotic and antithrombotic factors are elevated in patients with type 1 diabetes complicated by microalbuminuria. Diabetic Medicine 10: 122-128

25. Appel G, Blum B, Chien S, Kunis C, Appel A (1985) The hyperlipidaemia of the nephrotic syndrome: relation to plasma albumin concentration, oncotic pressure and viscosity. N Engl J Med 312: 1544-1548

26. Lam KSL, Pang RWC, Wat MS, Lauder IJ, Janus ED (1996) Apolipoprotein(a) levels and phenotypes in NIDDM patients with microalbuminuria and albuminuria. Nephrol Dial Transplant 11: 2229-2236

27. Ho CH, Wang SP, Jap TS (1995) Hemostatic risk factors of coronary artery disease in the Chinese. Int J Cardiol 51: $79-84$

28. de Sousa JC, Azevedo J, Soria C et al. (1988) Factor VII hyperactivity in acute myocardial thrombosis: a relation to the coagulation activation. Thromb Res 51: 165-173 